\title{
Evaluation of Carcinogenic/Co-carcinogenic Activity of Chikusaku-eki, a Bamboo Charcoal By-product Used as a Folk Remedy, in BALB/c 3 T3 Cells
}

\author{
Yuki Kimura, Shiho Suto, and Masaaki TATsuKA* \\ Department of Molecular Radiobiology, Research Institute for Radiation Biology and Medicine, Hiroshima University; \\ 1-2-3 Kasumi, Minami-ku, Hiroshima 734-8553, Japan. Received February 25, 2002; accepted May 17, 2002
}

\begin{abstract}
Chikusaku-eki is an acidic dark brown liquid obtained as a by-product from bamboo charcoal burners. The solution diluted with water is gaining widespread popularity in Japan as a folk medicine for skin diseases such as scabies, eczema, and atopic dermatitis. In this study, the carcinogenic and tumor-promoting potential of chikusaku-eki was determined using the BALB/c 3T3 A31-1-1 cell transformation system. Carcinogenic activity was tested by treating A31-1-1 cells for $24 \mathrm{~h}$ with $0.06 \%$ solution, a dose resulting in $35 \%$ clonogenic cell survival. In both 2-O-tetradecanoylphorbol-13-acetate (TPA)-treated and non-treated groups, chikusaku-eki did not initiate carsinogenesis. Following initiation with 3-methylcholanthrene (3-MCA), A31-1-1 cells were chronically treated with a non-toxic concentration range of chikusaku-eki $(\leq 0.01 \%)$, but chikusaku-eki did not act as a tumor promoter. Thus, chikusaku-eki was not carcinogenic/co-carcinogenic in the in vitro cell transformation assay examined in this study after being diluted more than $10^{4}$-fold with water.
\end{abstract}

Key words chikusaku-eki; bamboo; cell transformation; carcinogen; initiation; tumor promotion

Among a number of short-term evaluation systems for the detection of environmental carcinogens/mutagens, mammalian cell transformation systems are particularly useful because their assay endpoint is neoplastic conversion of target cells. BALB/c 3T3 A31-1-1 cells have been widely used to evaluate the carcinogenic/co-carcinogenic activity of various physical and chemical agents. ${ }^{1}$ This cell system can be used to detect carcinogens and tumor promoters, but as well to assess the anticarcinogenic effects of certain agents. ${ }^{2)}$

Chikusaku-eki and mokusaku-eki are widely used in Japan at the present time. Both are acidic dark brown liquid byproducts of the charcoal burner, however, each is produced from a different source; chikusaku-eki is from bamboo species, such as Phyllostachys pubescens or Phyllostachys bambusoides, and mokusaku-eki is from the wood of broadleaved trees such as Quercus serrata, Castanea crenata and Prunus jamasakura. Chikusaku-eki and mokusaku-eki are frequently diluted with water and mixed into the bath as folk remedy for scabies, eczema, atopic dermatitis, and other skin diseases. Despite a lack of scientific evaluation of their efficacy, both solutions are gaining widespread popularity in Japan.

The main component of both chikusaku-eki and mokusakueki is acetic acid. In addition, both contain more than 200 accessory ingredients, including phenolic compounds, polyphenolic compounds, and various organic acids. Some of these ingredients, such as guaiacol, cresol, 4-ethyl-2-methoxyphenol, and 2,6-dimethoxyphenol, have been reported to have antifungal activity. ${ }^{3)}$ However, both solutions also contain chemical carcinogens such as woodcreosote, benzo[a]pyrene, benz[a]anthracene, and 3-methylcholanthrene (3-MCA). Unfortunately, no information exists with regard to the neoplastic potential of either solution. In the present study, we investigated the carcinogenic and tumor-promoting activity of chikusaku-eki in the BALB/c 3 T3 cell transformation system, using A31-1-1 target cells.

\section{MATERIALS AND METHODS}

Cells and Culture BALB/c 3T3 A31-1-1 cells were used as target cells for the cell transformation assay. ${ }^{4}$ Using this system, carcinogenic/co-carcinogenic activity was measured quantitatively. Cells that had been passaged only a few times were used for the transformation assay. The cells were cultured in Eagle's minimal essential medium supplemented with 10\% fetal bovine serum (Lot No. SF70521, Bocknek Laboratories, Etobicoke, Ontario, Canada) in $5 \% \mathrm{CO}_{2}$ at $37^{\circ} \mathrm{C}$. All transformation experiments were performed using the same lot of serum.

Chemicals Two known chemical carcinogens, 3-MCA, which is an initiator, and 2-O-tetradecanoylphorbol-13-acetate (TPA), which is a tumor promoter, were purchased from Sigma (Sigma-Aldrich Japan, Tokyo). The chosen brand of chikusaku-eki for this experiment was based on the recommendation of the Japan Bamboo Charcoal and Bamboo Vinegar Association (Kyoto). The Association guaranteed the quality of the brand used in this study, which was produced by the Obama Take-Sumi Production Association, Obama, Fukui. This chikusaku-eki was prepared from Phyllostachys pubescens. The solution was translucent and dark brown in colour and was acidic ( $\mathrm{pH}$ 3.0). However, once the solution was diluted 100 -fold with culture medium ( $\leq 1 \%$ solution), it was not acidic and a neutral culture $\mathrm{pH}$ was obtained.

Clonogenic Survival Assay To evaluate the cytotoxicity of chikusaku-eki on cultured cells, a clonogenic survival assay was performed. Exponentially growing cells (100) were seeded into $60-\mathrm{mm}$ culture dishes (Nalgen Nunc International, Tokyo) with $4 \mathrm{ml}$ of culture medium. After $24 \mathrm{~h}$, the culture medium was replaced with fresh medium containing various concentrations of chikusaku-eki. To test the acute effects of exposure, the chikusaku-eki medium was again replaced with fresh medium after $24 \mathrm{~h}$, and the cells were cultured for another $5 \mathrm{~d}$ to allow colonies to form. To test the cytotoxic effects of chronic exposure, the cells were cultured with chikusaku-eki-containing medium for a further $6 \mathrm{~d}$ after 
the initial $24 \mathrm{~h}$ culture in fresh medium, during which time colony formation took place. The colonies that appeared were stained with Giemsa solution and counted under a stereomicroscope. Cells within colonies of more than 50 cells were counted as surviving cells.

In Vitro Transformation Aassay Exponentially growing cells $\left(10^{4}\right)$ were seeded into $60-\mathrm{mm}$ culture dishes with $4 \mathrm{ml}$ of culture medium. To evaluate the initiating activity of chikusaku-eki, transformation was initiated $24 \mathrm{~h}$ later with either $0.06 \%$ chikusaku-eki or $2.5 \mu \mathrm{g} / \mathrm{ml} 3-\mathrm{MCA}$ as a positive control. After the initiation period of exposure to chikusakueki or 3-MCA for $24 \mathrm{~h}$, cells were cultured in fresh medium for another $5 \mathrm{~d}$. After $5 \mathrm{~d}$, the culture was again replaced with either fresh medium or fresh medium containing $100 \mathrm{ng} / \mathrm{ml}$ TPA, which was changed twice weekly. The TPA-treated cells were cultured in this manner with TPA-containing medium for two weeks, after which they were placed in fresh medium which was changed twice weekly. To evaluate the promoting activity of chikusaku-eki, transformation was initiated $24 \mathrm{~h}$ later with $2.5 \mu \mathrm{g} / \mathrm{ml} 3$-MCA. After an initiation period with $3-\mathrm{MCA}$ for $24 \mathrm{~h}$, the cells were cultured in growth medium for $5 \mathrm{~d}$. Then, the culture was replaced with fresh medium or fresh medium containing chikusaku-eki $(0.01-0.0001 \%)$, which was changed twice weekly. After two weeks of exposure, the culture medium of chikusakueki-treated cells was again replaced with fresh medium and changed twice weekly. Thirty days after initiation of the experiment, all cultures were fixed and stained with Giemsa. Morphological transformation was determined under a dissecting microscope. Only densely stained multilayer foci with criss-crossing of cells at their periphery were scored.

\section{RESULTS}

Cytotoxic Effects of Chikusaku-eki on Cultured Cells Chikusaku-eki is acidic, but is neutral in culture, having been diluted 100-fold with culture medium. Thus, $\mathrm{pH}$ of chikusaku-eki medium was thought to be not toxic. Nevertheless, chikusaku-eki contains cytotoxic compounds such as wood tar derivatives. We first, therefore, tested the cytotoxic effects of chikusaku-eki on BALB/c 3T3 A31-1-1 cells. Two different treatment schedules, $24 \mathrm{~h}$ treatment and chronic exposure, were examined to determine the effects of chikusakueki during the initiation and promotion periods of experimentally-induced carcinogenesis. Figure 1A shows the survival rate of cells after 24-h exposure to chikusaku-eki, as determined by clonogenic assay. From these data, an applied dose for testing the initiation activity of chikusaku-eki on the cell transformation assay was determined as $0.06 \%$, which is a dose at $35 \%$ for clonogenic cell survival because initiation activity of carcinogens can be efficiently detected at a dose resulting in 35\% clonogenic cell survival. ${ }^{1)}$ We next examined the potential for cytotoxicity with chronic exposure to chikusaku-eki. The cells were seeded in normal growth medium and cultured to form colonies in medium containing $0.1 \%$ or lower concentrations of chikusaku-eki. All of the cells died in $0.1 \%$ chikusaku-eki culture (Fig. 1B). Thus, to evaluate promotion activity, $0.01 \%$ or lower concentrations of chikusaku-eki were used.

Carcinogenic/Co-carcinogenic Effects of Chikusaku-eki on Cell Transformation To establish whether chikusaku-
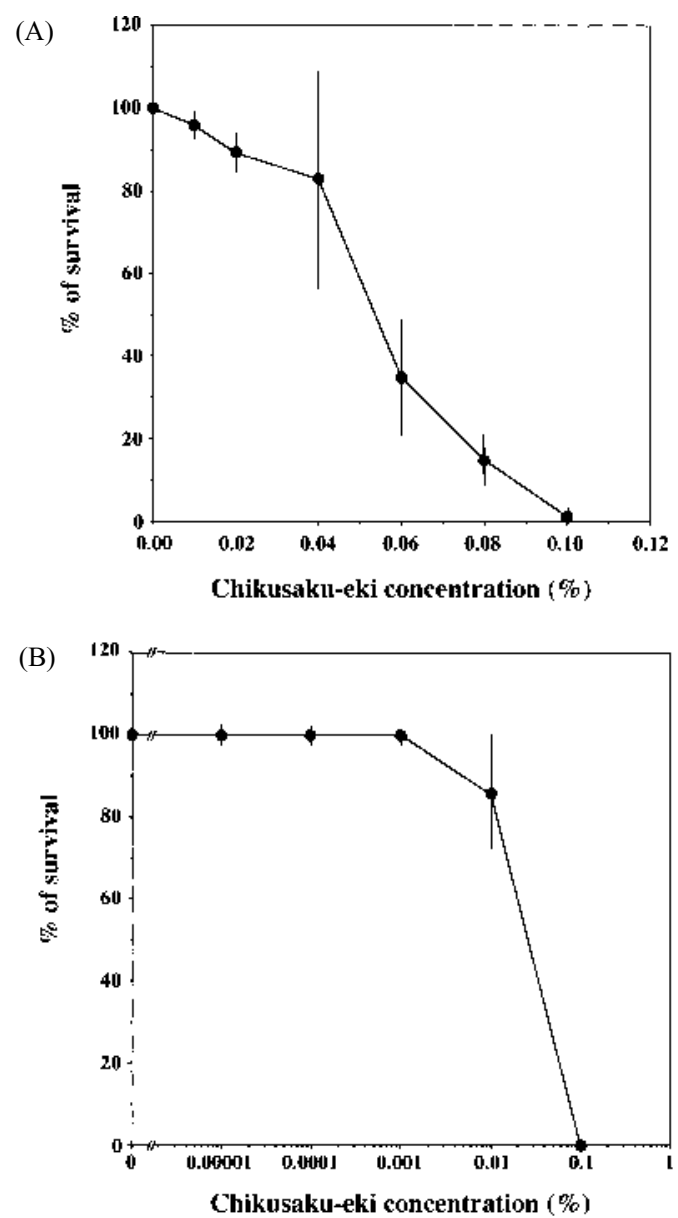

Fig. 1. Effect of Chikusaku-eki on the Colony Forming Ability of BALB/c 3T3 A31-1-1 Cells

Cells were seeded and cultured for $24 \mathrm{~h}$. The growing cells were treated with various concentrations of chikusaku-eki for $24 \mathrm{~h}$ (A) or $6 \mathrm{~d}$ (B). Cells within colonies containing over 50 cells were evaluated as surviving cells. Data points represent the mean of three independent experiments. Bars, S.D.

eki has carcinogenic/co-carcinogenic effects on cell transformation, we used a two-stage transformation system with BALB/c 3T3 A31-1-1 cells. The utilization of this system allowed us to evaluate the effects of chikusaku-eki on two distinct stages of carcinogenesis: 'initiation' and 'promotion'. To assess initiation activity, $0.06 \%$ chikusaku-eki was applied. As a positive control, $2.5 \mu \mathrm{g} / \mathrm{ml} 3-\mathrm{MCA}$ was used. To enhance initiating activity, $100 \mathrm{ng} / \mathrm{ml}$ TPA was used as a tumor promoter. The treatment schedule is shown in Fig. 2A. In both the 3-MCA-treated group and the 3-MCA plus TPAtreated group, transformed foci were observed (Fig. 2B, lanes $2,5)$. However, chikusaku-eki showed no initiating activity, even when TPA was applied as a tumor promoter (Fig. 2B lanes 3,6). Following this, the effect of chikusaku-eki was tested with regard to promotion of cell transformation (Fig. 3A). 3-MCA-initiated cells were chronically treated with $0.01 \%$ or less of chikusaku-eki. The transformation frequency of each chikusaku-eki-treated group was not different from that of the control group treated with 3-MCA alone (Fig. 3B, lanes 2, 4-6). This indicates that chikusaku-eki had no carcinogenic/co-carcinogenic effects on cell transformation at the concentration range applied in this study. 
(A)
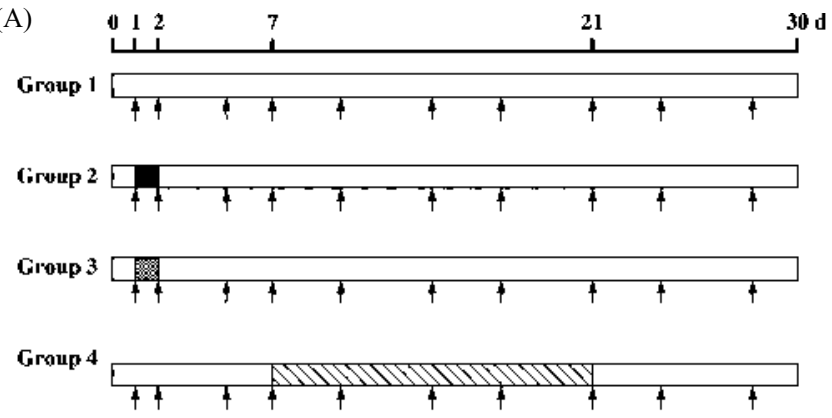

Group 5

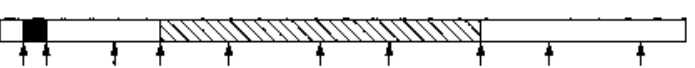

Gromp f

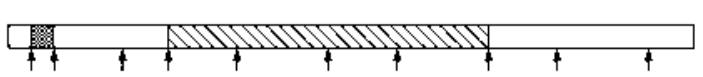

(B) Group Treatment

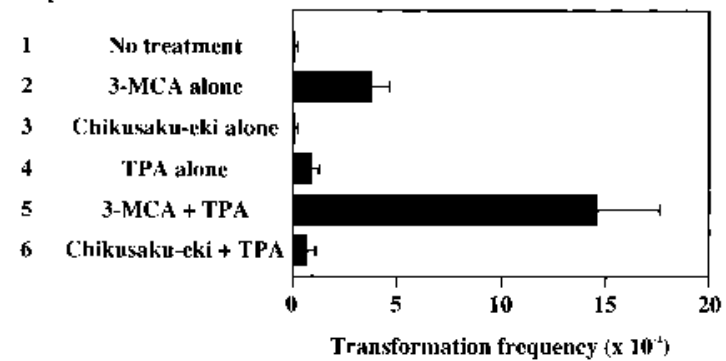

Fig. 2. Effect of Chikusaku-eki on Initiation of Cell Transformation Using BALB/c 3T3 A31-1-1 Cells

(A) The experimental protocol is illustrated. The target cells $\left(1 \times 10^{4}\right)$ were initiated with $2.5 \mu \mathrm{g} / \mathrm{ml} 3$-MCA ( $)$, or $0.06 \%$ chikusaku-eki (潭), for $24 \mathrm{~h}$. The initiated cells were promoted with $100 \mathrm{ng} / \mathrm{ml}$ TPA ( $($ ) for 2 weeks. $\uparrow$, indicates medium change. (B) Densely stained multilayer foci with criss-crossing of cells at their periphery were scored and the transformation frequency per surviving cell was calculated. The results were confirmed by two independent experiments. The differences between groups 1 and 3 and between groups 4 and 6 were not significant, according to the two-tailed MannWhitney $U$ test. Data present the mean of one representative experiment in which ten dishes were used. Bars, S.D.

\section{DISCUSSION}

Both chikusaku-eki and mokusaku-eki have recently gained widespread popularity in Japan, particularly among patients looking for folk remedies for the treatment of scabies, eczema, atopic dermatitis, and other skin diseases. Despite their popularity, their carcinogenic/co-carcinogenic activity has not been evaluated. In this study, the same batch of high-quality chikusaku-eki was used to avoid possible batchto-batch or brand-to-brand variation in chemical constituents. The quality of this brand was guaranteed by the Japan Bamboo Charcoal and Bamboo Vinegar Association. The brand of chikusaku-eki used is known to contain benzo[a]pyrene, benz[a]anthracene, and 3-MCA at $\mathrm{ng} / \mathrm{ml}$ levels or less. These carcinogens are present at levels below the regulatory limits of the Resource Conservation and Recovery Act of United States of America for the disposal of hazardous waste. Concentrations below these levels are considered safe with regard to their risk of promoting cancer in humans. However, apart from these carcinogens, chikusaku-eki also contains various known and unknown tar-derivatives. Therefore, it is necessary to evaluate its carcinogenic/co-carcinogenic activity. We did so and found that chikusaku-eki is not carcinogenic/co-carcinogenic in the BALB/c $3 \mathrm{~T} 3$ cell transformation system. To our knowledge, this is the first experiment to

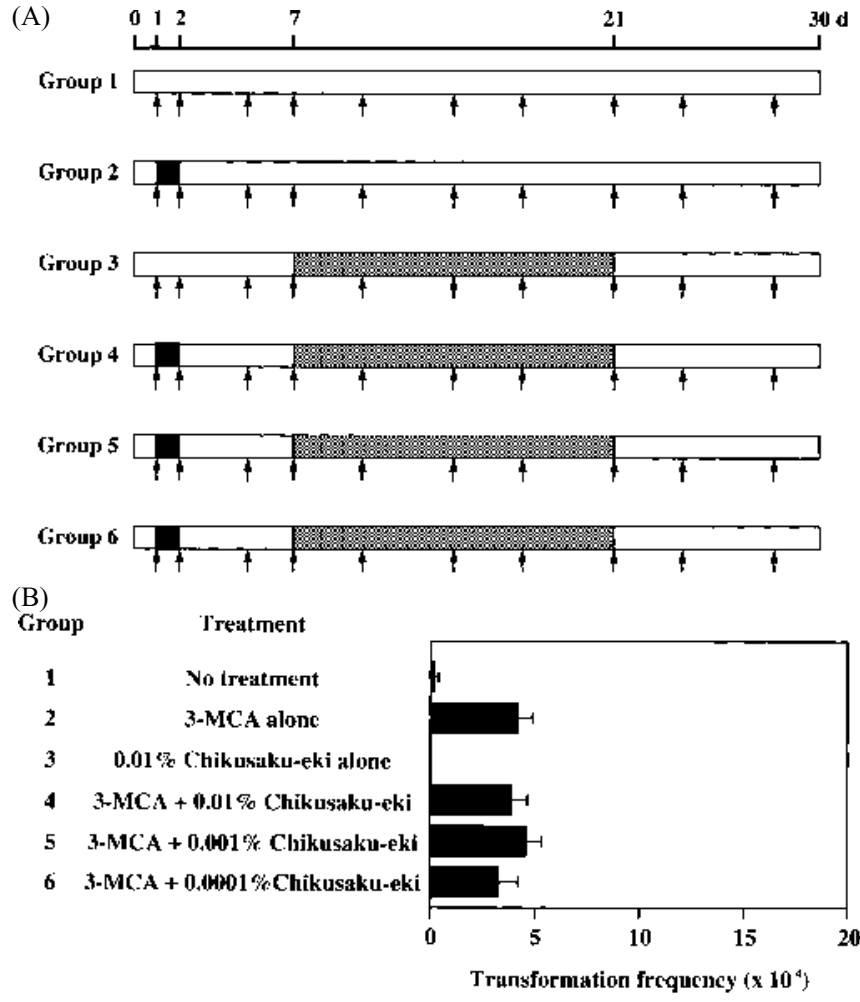

Fig. 3. Effect of Chikusaku-eki on Promotion of Cell Transformation Using BALB/c 3T3 A31-1-1 Cells

(A) The experimental protocol is illustrated. The target cells $\left(1 \times 10^{4}\right)$ were initiated with $2.5 \mu \mathrm{g} / \mathrm{ml} 3-\mathrm{MCA}$ ( $\square$ ) for $24 \mathrm{~h}$. The initiated cells were promoted with various concentrations of chikusaku-eki (鳓) for 2 weeks. $\uparrow$, indicates medium change. (B) Densely stained multilayer foci with criss-crossing of cells at their periphery were scored and the transformation frequency per surviving cell was calculated. The results were confirmed by two independent experiments. The differences between groups 1 and 3 , between groups 2 and 4, between groups 2 and 5, and between groups 2 and 6 , were not significant according to the two-tailed Mann-Whitney $U$ test. Data represent the mean of one representative experiment in which ten dishes were used. Bars, S.D.

investigate its effects even in single brand.

Chikusaku-eki is usually diluted with water for use in the bath. In Japan, bathtubs in the home hold more than 500 liters. In general, 5-50 $\mathrm{ml}$ of chikusaku-eki or mokusakueki are added to bath water. This results in a concentration range of $0.01-0.001 \%$. It follows that the skin and mucous membranes of the human body are exposed to chikusaku-eki at this concentration range during bathing. From the data presented here, it can be suggested that chikusaku-eki is not carcinogenic/co-carcinogenic. In addition, although chikusakueki did not significantly suppress the initiation activity of 3MCA when applied during the promotion period of the cell transformation assay, it did show a tendency to reduce the frequency of spontaneous transformation (Fig. 3). To determine the potential anti-carcinogenic effects of chikusaku-eki, further evaluation is needed to examine the effects of different treatment schedules and their effects on other models of carcinogenesis.

Chikusaku-eki and mokusaku-eki are similar, but chikusaku-eki is generally recognized to have greater anti-inflammatory activity than mokusaku-eki. The variation in their composition stems from the fact that they come from different raw materials. For both liquids, it is necessary to elucidate their active ingredients and their efficacy, but evaluation of their safety is undoubtedly a more pressing concern 
given their widespread use.

Acknowledgements This research was partially supported by the Ministry of Education, Science, Sports and Culture, Grant-in-Aid for Scientific Research on Priority Areas (Cancer), 13214070, 2001. We thank Kayo TakeiNagano and Kazue Nakakura for their assistance with the preparation of this manuscript, Takahide Ota for his helpful discussion, and Fumio Suzuki for his encouragement throughout this project.

\section{REFERENCES}

1) Kakunaga T., Yamasaki H. (ed.), "Transformation Assay of Established Cell Lines: Mechanisms and Application," No. 67, IARC Scientific Publications, Lyon, 1985.

2) Tatsuka M., Maeda M., Ota T., Jpn. J. Cancer Res., 92, 1184-1189 (2001).

3) Ikegami F., Sekine T., Fujii Y., Yakugaku Zasshi, 118, 27-30 (1998).

4) Kakunaga T., Crow J. D., Science, 209, 505-507 (1980). 